\title{
Inaugural Issue Editorial
}

Welcome to this inaugural issue of ACM Transactions on Computing for Healthcare (HEALTH). We are excited to launch this new journal for an area that is growing in importance but that has yet had no top-quality journal dedicated to computing professionals working in healthcare. We are very proud to be the founding Editors-in-Chief and hope that the community will contribute to its success by submitting their best work, and by performing timely and substantive reviews.

Computing for healthcare has emerged as an important and growing research area. By using smart devices, the Internet of Things for health, mobile computing, machine learning, cloud computing, and other computing-based technologies, computing for healthcare can improve the effectiveness, efficiency, privacy, safety, and security of healthcare (e.g., personalized healthcare, preventive healthcare, ICU without walls, and home hospitals). The goal for HEALTH is to become the premier publication for high-quality original research papers, survey papers, and challenge papers with scientific and technological results pertaining to how computing is improving healthcare. This journal is multidisciplinary, intersecting computer science, data science, electrical and computer engineering, mechanical engineering, bio-medical engineering, behavioral and social science, psychology, and the health field in general. However, all submissions must show evidence of their contributions to the computing field as informed by healthcare. We will not publish papers on large pilot studies, diseases, or other medical assessments/results that do not have novel computing research results.

Many people have contributed to the creation and launch of HEALTH. From the ACM, we thank the Publications Board for encouraging us to develop this journal, and we are grateful to Laura Lander and Victoria White for their incredible help! We thank the executive advisory board members for their guidance: Debra Estrin, William Kaiser, and Rosalind Picard. We are also fortunate to have a very strong collection of associate editors, and we especially thank them for their willingness to process submitted papers. We would also like to thank David Kotz and Gouliang Xing for running our first special issue on Wearable Technologies for Smart Health-a future issue. Last, we would like to thank our information director, Meiyi Ma, a graduate student at the University of Virginia, for her excellent and timely help.

This first issue consists of five very interesting articles, including three invited papers, on a variety of important topics, including wearable devices for health, modeling of medical data, providing recommendations, monitoring the deterioration of outpatients, and a clinical decision assistant system. It is our hope that you enjoy reading them.

John A. Stankovic BP America Professor University of Virginia

Insup Lee

Cecilia Fitler Moore Professor University of Pennsylvania

Editors-in-Chief

(C) 2020 Copyright held by the owner/author(s).

2637-8051/2020/02-ART1

https://doi.org/10.1145/3375711 